\title{
Reform of the Web Design and Construction Teaching
}

\author{
Haibo Wang \\ Computer and Network Center \\ Communication University of China \\ Beijing, China \\ wanghb@cuc.edu.cn
}

\author{
Weina Zhang \\ Computer and Network Center \\ Communication University of China \\ Beijing, China \\ zhangweina@cuc.edu.cn
}

\begin{abstract}
This paper puts forward reform method for the web design and construction teaching from three aspects. Based on computational thinking, we redesigned guiding principle in HTML element and CSS layout teaching, emphasized on abstraction and automation. Establish a real development environment of website which can make students comprehend the real process in the website building. Adopted more tools for analyze web page which provide powerful function to students. These tools help students to acquire experience from mature web page. These reforms have been proved to be effective in web design and construction teaching.
\end{abstract}

Keywords- Web design and construction; computational thinking; layout; content-box model; Firebug; Web developer

\section{INTRODUCTION}

With the development of Internet, web page has been the main media for modern information. Mastering the basic knowledge of web design and construction will help students to use Internet more effectively. The main content of the course of web design and construction include the components of web page, layout of web page and style of web page. Further, it also includes the whole construction procedure of website, such as link between web page, upload and update of website, etc.

For a normal student in university, it isn't unacquainted for them to use browser surfing on the Internet. But the essence behind the procedure is uncomprehending to them, such as the communication process between the browser and the website server, the components of the web pages, the layout principle of web page, etc. We conducted reforms in three aspects in order to improve the teaching effect.

\section{REFORM OF GUIDING PRINCIPLE BASED ON COMPUTATIONAL THINKING}

In March 2006, Professor Jeannette M. Wing of Carnegie Mellon University promoted the concept of "Computational Thinking” at Communications of the ACM firstly [1]. She pointed out that computational thinking is a fundamental skill for everyone, not just for computer scientists. She also indicated that computational thinking involves solving problems, designing systems, and understanding human behavior, by drawing on the concepts fundamental to computer science.

\section{A. HTML element teaching}

At first sight, people feel a web page from the visual elements, such as color, layout, letter, images, etc. For web page designer, he must abstract the visual element of the web page to abstract tag using HTML. Further, through Cascading Style Sheet, web page designer can control the style of the tag. The ability of abstraction is the basic quality of a web page designer. Without the rational abstraction, web page will be constructed with wrong structure.

Under the guide of computational thinking, besides the basic knowledge of the meaning of tag, we emphasize the ability of discriminating the elements on web page. For example, in Fig 1, we trained the students to recognize the components of web page, and translate them to abstract tag.

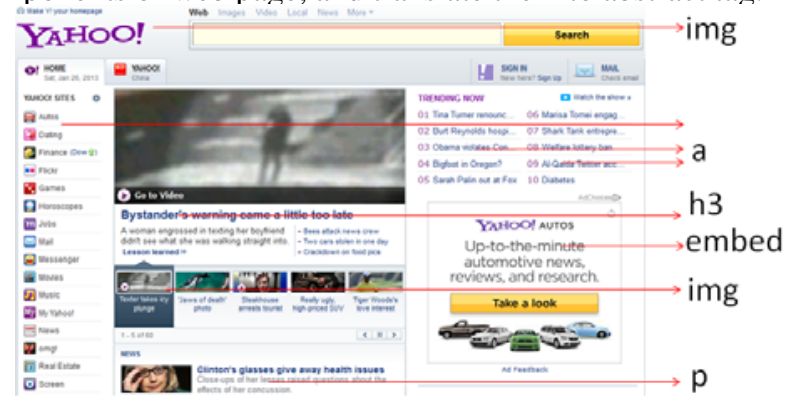

Figure 1. From visual element to abstract HTML tag

\section{B. Layout of web page teaching}

Besides acquiring web page from website server, the main function of browser is to put every element of web page on the right place, namely realize layout of web page. In the realization process, the browser treats every element as a rectangle. To design web page without obstacle, students must master the essence of layout of web page. This can be summarized by two keywords from computational thinking: abstraction and automation.

When the web page is invented, the layout of web page is realized with the help of table. The elements of web page are placed in the data cell of table. It can be regarded as place book, vase, etc. on a bookcase.

Currently, the layout method of web page is replaced with DIV plus CSS [2] [3] [4]. The main idea behind this is to separate style from structure. In HTML file, tag is used to represent the structure of the web page, such as logo, navigation bar, main body, copyright, etc. In CSS file, layout 
of web page and style for the elements on the web page is described.

The main principle of this is called "content-box model", which is showed in Fig 2. Every element on web page is regarded as a box. Each box has content, padding, border and margin. Through setting up these aspects, web designer can control elements on the web page in detail, such as setting up the width of web page, remaining 10px spaces between main body and navigation bar, etc.

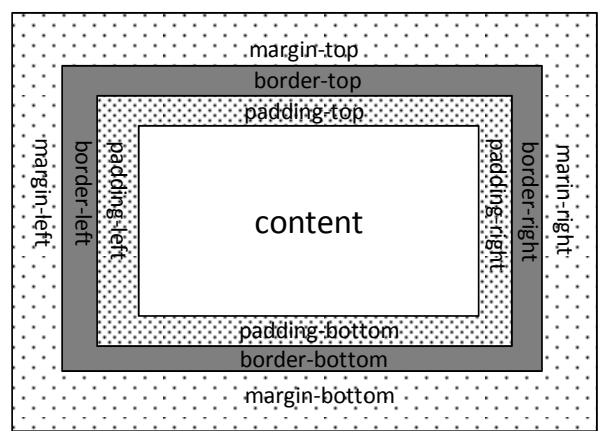

Figure 2. CSS content-box model

In teaching of content-box model, we draw support from computational thinking for the interpretation of the relevant conception. To make the conception intelligible, we demonstrated the model by using animation of balloon in Fig 3. Each balloon represents a div element in web page. Before the float property is set up, each balloon occupies a single line. Once setting up the float property of the first balloon to "left", the right space of the first balloon will be released. Then the below balloon will move to the right side of the first balloon. Setting up the same property of the other balloons, they will all move to the first line until the whole width of balloon container isn't enough for the summation of the balloon width.
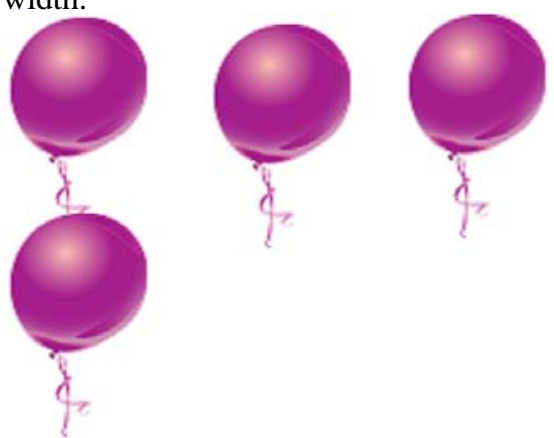

Figure 3. Animation of balloon illustraing layout of web page

\section{REFORM OF DEVELOPMENT ENVIRONMENT OF WEBSITE}

In general cases, students do exercises of web page on the laboratory course. Further, they submit their web page homework through attaching file on the teaching management system. This pattern has two weaknesses: on the one hand, it isn't convenient for students to share experience with each other; on the other hand, the students can't comprehend the real process in the website building.

To solve this problem, we build a real development environment which is same as professional website for students. We adopted FileZilla Server [5] as our FTP server. Every student is assigned an account on the server and a folder for uploading web page. We use Apache HTTP server as Web server. After students upload their homework through FTP client, they can see their web page on web server, just like the real process in website construction. This process is showed in Fig 4.

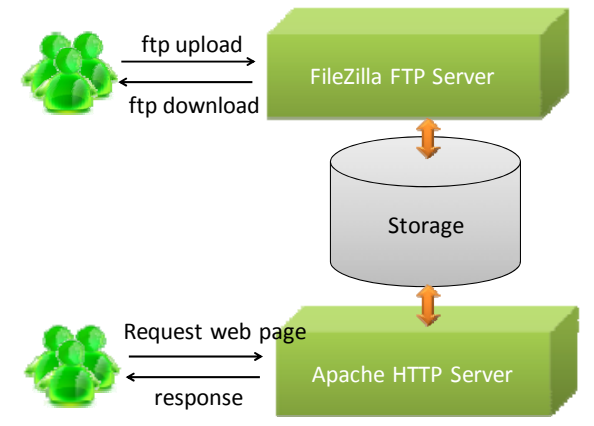

Figure 4. Real website development environment

Due to the huge number of students, there is the need to create student accounts in bulk. FileZilla server doesn't support this function itself. Through analyzing the configuration file of FileZilla server, we can find that the account information is stored in a file named "FileZilla Server.xml". For one user account, the basic information is showed in Fig 5:

$$
\begin{aligned}
& 1<\text { User Name="u1"> } \\
& 2<\text { Option Name="Pass" }>x x x</ \text { Option }> \\
& 3<\text { Option Name="Group" }>\text { xx }</ \text { Option }> \\
& 4 \quad<\text { Permissions> } \\
& 5<\text { <ermission Dir=" } x x x x x \text { "> } \\
& 6<\text { Option Name="DirCreate" }>1</ \text { Option }> \\
& 7<\text { Option Name="FileDelete" }>1<\text { /Option }> \\
& 8</ \text { Permission }> \\
& 9<\text { Permissions }> \\
& 10</ \text { User }>
\end{aligned}
$$

Figure 5. FileZilla user account

The meanings of each line are as follows:

1: user configuration begin and the name of the user

2: the password of user which is encrypted

3: the group the used belongs to

4: permission configuration region begin

5: directory allowed for the user on the ftp server

6: permission for create directory

7: permission for delete file

8: permission configuration end

9: permission configuration region end

10: user configuration end 
Based on these principles, we developed a tool to generate the XML configuration for massive students in batch mode based on the student ID. After using the created XML file to replace the default configuration file, we created accounts for every student.

\section{REFORM OF TOOLS FOR WEB PAGE DESIGN}

In the process of web design and construction, the normal tools for editing web page can be classified to two kinds: non-visual tools and visual tools. The typical non-visual editing tools include EditPlus, UltraEdit, Notepad++, etc. For visual editing tools, Dreamweaver is used by web page designer widely. We try to adopt more tools to promote the efficiency of the students.

\section{A. Tool for examine the size of web page}

For the size of Word document, most students are familiar with A3, A4, B5, etc. For web page, the conception is different. The first conception web page designer should know is resolution of display. The same web page will be presented differently on website visitor's browser with different resolution. With the development of display technology, the resolution of display advanced. It varied from the early $640 * 480,800 * 600$, to the current $1024 * 768$, $1440 * 900,1600 * 900$, etc. In the Word, it provides rule which be used to view the document size in detail. For browser, it doesn't provide the same function. We adopted a tool named FastStone Capture [6]. Besides the function of screenshot, it provides a virtual rule which can be placed on top of web page. Through this rule, we can measure the actual size of the whole page or a part of web page, Fig 6 shows that the width of tech channel of Sina website is 950px.

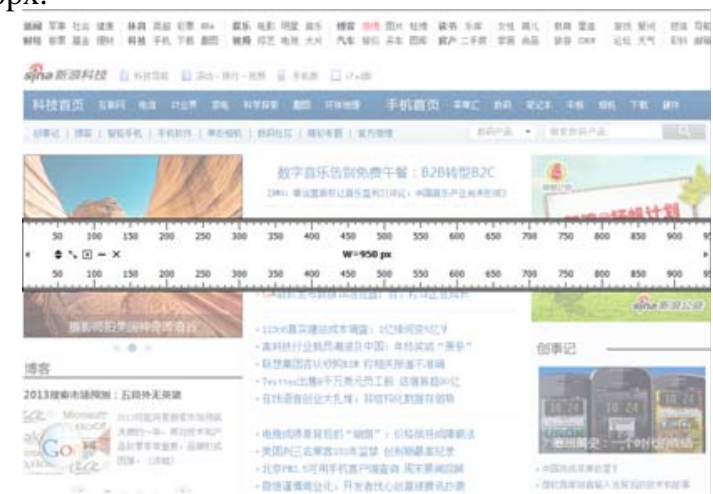

Figure 6. Examine size of web page by FastStone Capture

\section{B. Tools for examine the structure,style and content-box model of specific element}

The HTML source code of web page can be seen by the user once the web page is downloaded and showed on the browser. This characteristic provides convenience for the web page designer to absorb experience from the other website. With the web page get more and more complicated, it became difficult for web page designer to understand and position a specific element on the web page. We adopted a tool named "Firebug", which is a plugin for Firefox browser
[7]. It provides powerful function for user to analyze web page. If the user wants to examine a specific element in page, firstly click the "Inspect" button, secondly moves the mouse above the web page, the current element under mouse is highlighted with box. If the user clicks the left key of mouse, the tag and the style of selected elements will be showed on the panel of Firefox.

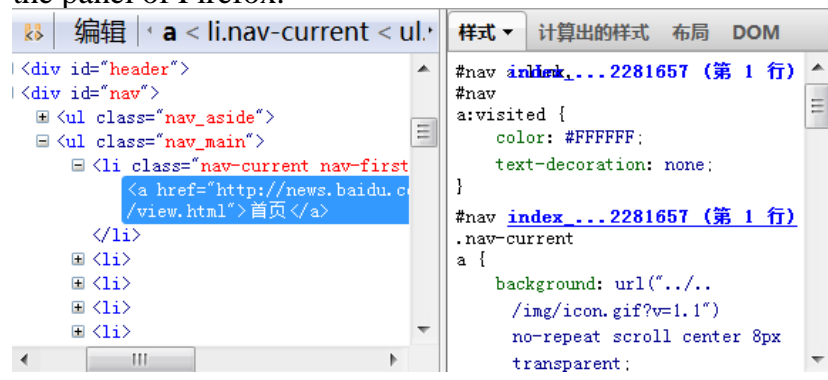

Figure 7. Examine structure and style of a specific element

As showed on the left side of Fig 7, the element in the navigation bar is using <li $>$ tag to become an item in an unordered list. Within the list item, $<\mathrm{a}>$ tag forms a hyperlink. On the right side of Fig 7, it shows that the style of hyperlink. For example, It shows after the hyperlink is clicked, it will be showed with no underline and white color style.

As discussed in front, the content-box model is a very important conception in web page design. Examine the content-box model of specific element is helpful for the indepth understanding how the web page is constructed. We also use Firebug to get information about this aspect.

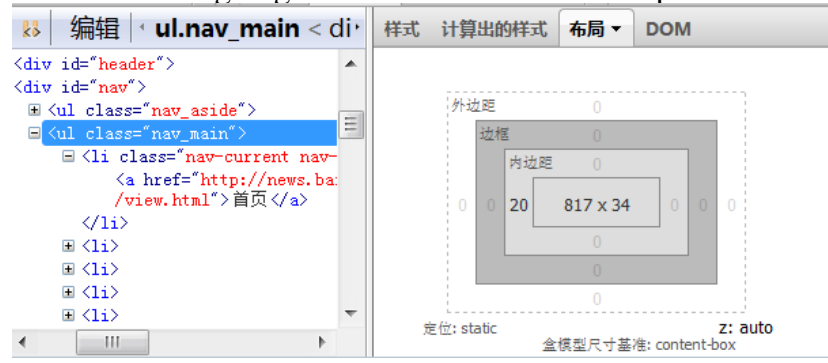

Figure 8. Examine content-box model using Firefox

As showed in left side of Fig 8, the current selected element for inspection is a $<\mathrm{ul}>$ tag which is used as main part of the navigation bar. On the right side of Fig 8, it shows different aspect of content-box model of this element. For example, the width and height is $817 \mathrm{px}$ and $34 \mathrm{px}$ respectively. The top, right and bottom padding is $0 \mathrm{px}$ while the left padding is 20px. The top, right, bottom and left margin are all 0px.

\section{Tools for examine specific type of HTML element and color used on the web page}

In order to examine a specific type of HTML element, we use a tool named "Web Developer", which is also a plugin for Firefox browser [8]. It provides lots of function to observe web page. For example, it can show the web page meanwhile disabled effects of the CSS on the web page. It 
can also mark all block level elements on the web page or custom elements which can be set up by user. Fig 9 shows all $<$ div $>$ elements on the web page. It is marked with rectangle in red color, which help user to understand the structure of web page in depth.

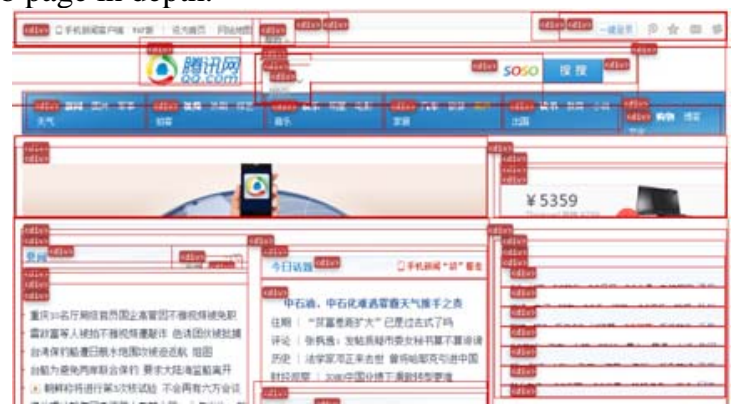

Figure 9. Examine Div element on a web page

"Web Developer" also provides the function to observe color used in a web page. For a novice in web design, it is difficult for them to think which color should be used. Observation and study from the mature website is a method which can help them grow up more quickly. Fig 10 shows the color used on a web page. The novice can learn from this to examine color and color schemes used in a web page.
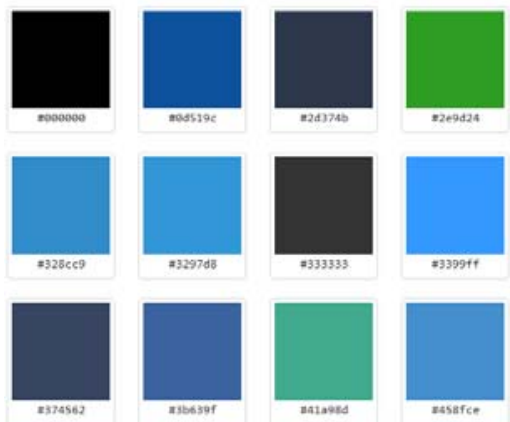

Figure 10. Examine color used in a web page

\section{Tools for examine the interactive process between the browser and the website}

Although the bowser is used by students almost every day, the process occurred behind the browser is a little unacquainted and mysterious for them. In fact, the browser uses HTTP protocol to communicate with website. For example, after the people input the URL of web page and press the return key, the browser will send "get" command with URL of web page as parameter to a website server. When the website server received this command, it will find the named web page from local disk. If the file is found, the website server will send it back to the user with a status code "200". If file isn't found, the website server will send back just a status code " 404 ". Based on the different response from the website server, the browser will act respectively. If the web page is acquired successfully, it will render the web page on the screen according to the CSS of the web page.

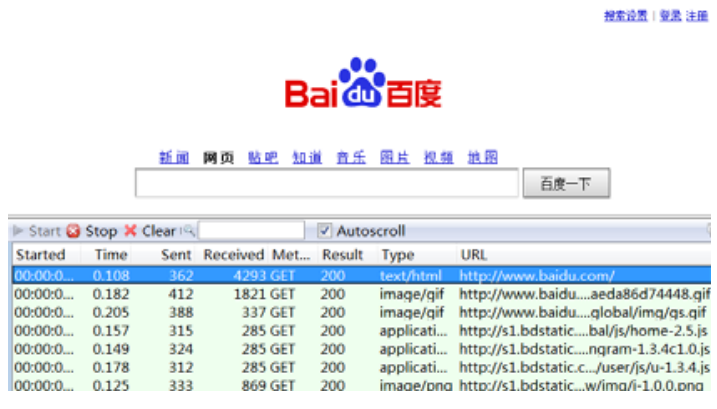

Figure 11. Interactive process between the browser and the website

In order to make these processes explicated, we adopted a tool named "Httpfox" which is also a plugin for Firefox [9] browser. Fig 11 shows the interactive process when the browser visits the Baidu website. The URL of the first page the browser gets from website server is "http://www.baidu.com". Httpfox shows that this web page is acquired with response code "200", which means success. It also shows this web page 4293 byte. After this web page is acquired, the browser analyze the web page to abstract other media files embedded on the web page, such as image file, JavaScript file, CSS file, etc. Then the browser makes more requests to the website server to acquire these files. Fig 11 shows the other request from line 2, which also include the response code, file size, URL, etc.

\section{CONCLUSION}

Web design and construction is a comprehensive course founded in many universities. Complex knowledge makes the course not easy to be mastered. In this paper, we reform web design and construction teaching from three aspects. Web redesigned guiding principle of course based on computational thinking, emphasized on abstraction and automation. We built development environment same as the real website, which can make students experience the real process in the website building. Through adopted more auxiliary tools, students can analyze web page easily which can help them master web design techniques more rapidly. It proved these reforms have produced better effects in web design and construction teaching.

\section{REFERENCES}

[1] Jeannette M. Wing, "Computational Thinking", communications of the ACM March Vol. 49, No. 3,pp. 33-35, 2006.

[2] A. Buddy, S. Collison, CSS mastery advanced web standards solutions, Posts \& Telecom press, 2010.

[3] D. Shea, M. E. Holzschlag, The Zen of CSS design, Posts \& Telecom press, 2012.

[4] E. A. Meyer, Smashing CSS professional techniques for modern layout, Posts \& Telecom press, 2012.

[5] FileZilla, http://filezilla-project.org/

[6] Faststone Capture, http://www.faststone.org

[7] Firebug, http://getfirebug.com

[8] Web developer, ttp://chrispederick.com/work/web-developer

[9] Httpfox, http://code.google.com/p/httpfox 